\title{
Information and Paternalism
}

Oren Bar-Gill

2015-01-08T07:41:39

Disclosure mandates are often considered to be the least paternalistic of all regulatory techniques. Indeed, information provision is believed to enhance both autonomy and efficiency by facilitating more informed decisionmaking. According to this traditional approach, disclosure regulation - a key instrument in the Nudge toolbox - is beyond reproach. Legitimacy concerns might be raised with respect to other Nudge-type interventions (specifically, the setting of default rules), but not disclosure.

I propose a two-pronged challenge to this conventional wisdom: (1) Behaviorallyinformed disclosure regulation is not (only) about information provision in the traditional sense; when we move beyond information provision, ethical questions start popping up. (2) Effective disclosure is necessarily selective, and the selection process raises paternalism/legitimacy concerns. This challenge is developed using examples from the regulation of consumer financial markets.

Behavioral economics has transformed disclosure. Disclosure is no longer about the provision of dry, objective information. Framing is crucial. How we disclose can be as important (or more important) than what we disclose. In the mortgage context, consider disclosing the risk of default and foreclosure in a way that invokes the loss frame. Pushing further away from the classical model, disclosure might not even be designed to assist the borrower's rational System 2 process. Rather, disclosure might be designed to influence decisions through the more intuitive System 1 process. (See Kahneman (2011), Bubb (2014).) Consider graphic warnings on cigarette labels. Or, back to mortgages, vivid descriptions or graphic portrayals of foreclosure. The conventional disclosure model envisions objective provision of information that will then be used by a rational decisionmaker to arrive at better outcomes. The new, behavioral disclosure paradigm severs or, at least, loosens the rational deliberation link between disclosure and outcome, suggesting an alternative channel - mediated by bias and emotion. When disclosure operates through this alternative channel, it deserves more serious scrutiny.

The second prong of the challenge concerns the selection of information to be disclosed. In many contexts, especially in the consumer finance space, decisions are complex and the amount of relevant information is staggering. If all this information is disclosed, consumers - even perfectly rational consumers - would ignore (most of) it. To avoid information overload, disclosure regulation must be selective. Faced with selective disclosure, sophisticated consumers draw rational inferences about undisclosed information. Less sophisticated consumers make biased inferences, or falsely assume that the partial disclosure contains all relevant information. For both groups of consumers, the selection of information for disclosure affects outcomes. And, accordingly, the selection should be subject to scrutiny. 
Consider the APR disclosure. The APR is supposed to provide a total cost of credit measure. But cost of credit is subject to interpretation. What fees are included and what fees are excluded? What formula should be used to aggregate different, temporally disparate cost dimensions? Should the APR figure account for the default option and the prepayment option? These regulatory choices carry significant implications. A loan's APR can vary substantially, depending on the regulator's answers to these questions.

Finally, the two prongs of the challenge interact in interesting ways. In particular, selection can be achieved by framing: the selected information can be prominently disclosed - in bold face, in large font, singled out in tabular form, whereas nonselected information can be hidden in the background - in the fine-print on the back of the form. The traditional, naïve approach mandates the disclosure of any piece of information deemed relevant, without regard to framing or formatting. Such disclosure may be ineffective, but it is less susceptible to manipulation. The new disclosure is more sophisticated and more nuanced. It is ushering a scientific approach to disclosure design. This behaviorally-informed disclosure is more effective, more powerful and, as a result, potentially more dangerous.

Disclosure is an important regulatory tool and it should remain a central option for regulators. Indeed, the new disclosure paradigm, based on behavioral economics, promises to produce better, more effective disclosure rules. There is a risk, however, that the no-scrutiny mindset, which was fine for the old, powerless disclosure paradigm, will be transferred to the new, powerful disclosure paradigm. The new disclosure should be celebrated. It should also be subject to careful study, and meaningful scrutiny.

References:

Kahneman, Daniel (2011), Thinking Fast and Slow.

Bubb, Ryan (forthcoming), TMI? Why the Optimal Architecture of Disclosure Remains TBD, Michigan Law Review.

(cc) $\mathrm{BY}-\mathrm{NC}-\mathrm{ND}$ 